\title{
AUTOMATIC BUILDING EXTRACTION USING A DECISION TREE OBJECT-BASED CLASSIFICATION ON JOINT USE OF AERIAL AND LIDAR DATA
}

\author{
R. Ghasemi Nejad ${ }^{1}$, P. Pahlavani ${ }^{1 *}$, B. Bigdeli ${ }^{2}$ \\ ${ }^{1}$ School of Surveying and Geospatial Engineering, College of Engineering, University of Tehran, Tehran, Iran - (r.ghasemi, \\ pahlavani)@ut.ac.ir \\ ${ }^{2}$ School of Civil Engineering, Shahrood University of Technology, Shahrood, Iran - bigdeli@ shahroodut.ac.ir
}

KEY WORDS: Building Extraction, Object-based, C4.5, Decision tree, Classification, Remote Sensing.

\begin{abstract}
:
Updating digital maps is a challenging task that has been considered for many years and the requirement of up-to-date urban maps is universal. One of the main procedures used in updating digital maps and spatial databases is building extraction which is an active research topic in remote sensing and object-based image analysis (OBIA). Since in building extraction field a full automatic system is not yet operational and cannot be implemented in a single step, experts are used to define classification rules based on a complex and subjective "trial-and-error" process. In this paper, a decision tree classification method called, C4.5, was adopted to construct an automatic model for building extraction based on the remote sensing data. In this method, a set of rules was derived automatically then a rule-based classification is applied to the remote sensing data include aerial and lidar images. The results of experiments showed that the obtained rules have exceptional predictive performance.
\end{abstract}

\section{INTRODUCTION}

Some of the previous researches in object extraction focused on pixel based image fusion and classification (Bigdeli et al. 2013, Bigdeli et al. 2016, Bigdeli et al. 2017, Pahlavani et al. 2017, Bigdeli et al. 2014). However, in recent decades, object-based image analysis (OBIA) create a new perspective in remote sensing image processing. Object-based classification is the approach that classifies no single pixels but groups of pixels called segments. In Object-based classification, remote sensing experts use a wide range of features to interpret images such as spectral information, texture, geometry, spatial relations, etc. However, the quality of a classification is not always increased by inserting a higher number of features (Bruzzone and Serpico 2000). Consequently, the quality of classification must be based on a subset of features identified due to their ability to recognize the classes and also depends on the classification method chosen by the expert. Traditionally, the classification is performed through the definition of rules by experts (e.g. a "Building" segment is a segment with a mean DSM value higher than a threshold). This process is usually done visually by an expert through a "trial-and-error" process and thresholds were derived empirically (Arvor, Saint-Geours et al. 2013). Therefore, the final accuracy of the classification depends too much on the expert knowledge and maybe two experts will define different rules for a same class and thus produce different maps. Therefore, the use of an automatic method to extract the rules seems essential.

From a decision tree model, the C4.5 decision tree algorithm, which is proven to be efficient, accurate and robust by many researches can produce a set of rules to make predictions (Ren, Zargham et al. 2006). With the aim of building extraction, this method is used in this paper to generate rules automatically from remote sensing data without expert intervention.

In the last decades, considerable attempts have been made to develop various methods for the detection of different types of objects in aerial and satellite images, especially buildings (Lhomme, He et al. 2009, Ahmadi, Zoej et al. 2010, Akçay and
Aksoy 2010, Benedek, Descombes et al. 2011, Stankov and He 2014, Sun, Pahlavani et al. 2017, Zhang et al. 2018, Xu, Wu et al. 2018, Shi, Mao et al. 2019). With the increasing availability and wide utilization of high resolution imagery, object-based image analysis (OBIA) has become a new approach or paradigm to classify or map satellite images into meaningful objects (Teo and Chen 2004, Walter 2004, Blaschke 2010, Pang, Hu et al. 2014, Toure, Stow et al. 2016, Li, Zhang et al. 2018, Gavankar and Ghosh 2019). OBIA rests upon two interrelated methodological pillars, i.e. (1) segmentation for nested, scaled representations; (2) rule-based classifiers for making explicit the required spectral and geometrical properties.

Image segmentation is the main step and a necessary prerequisite for extracting building blocks. Numerous image segmentation techniques have been developed and applied in remote sensing image analysis, such as (Baatz and Schäpe 2000, Benz, Hofmann et al. 2004, Blaschke, Burnett et al. 2004, Gao, Mas et al. 2011, Drăguţ, Csillik et al. 2014, Ming, Li et al. 2015, Chen, Zheng et al. 2018, Huang, Meng et al. 2019).

Some studies, use rule-based classification to extract objects but in many cases the experts are then used to define classification rules, based on a subjective process by advising which features to select and which rules to apply (Liu, Wang et al. 2005, Yu, Gong et al. 2006, Zhou, Troy et al. 2008, Bouziani, Goita et al. 2010). In order to achieve more robust results in OBIA, it is necessary to extract rules by automatic methods. In some studies, rule-based via automatic methods are applied. For example, (Ren, Zargham et al. 2006, Zhang and Zhu 2011, Jumlesha, Babu et al. 2012, Arvor, Saint-Geours et al. 2013, Ziaei, Pradhan et al. 2014).

\section{STUDY AREA AND DATA SELECTION}

The ISPRS benchmark dataset of Potsdam (Germany) is an open asset dataset.

\footnotetext{
* Corresponding author
} 


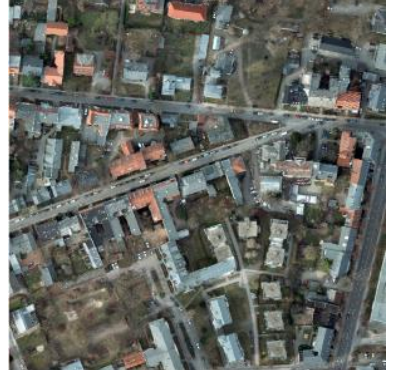

a) Aerial photograph

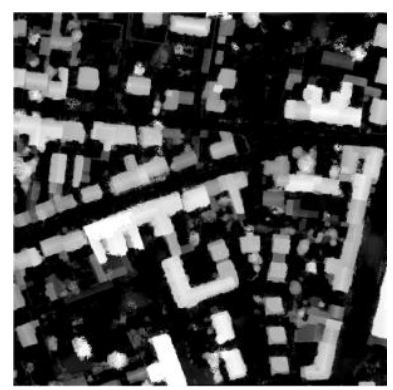

c) NDSM

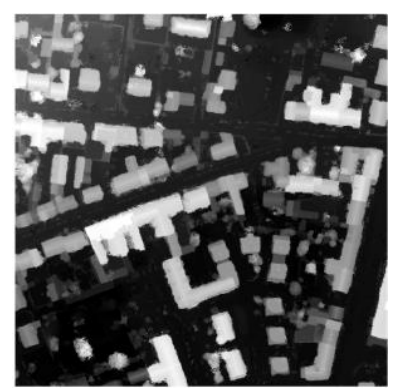

b) DSM

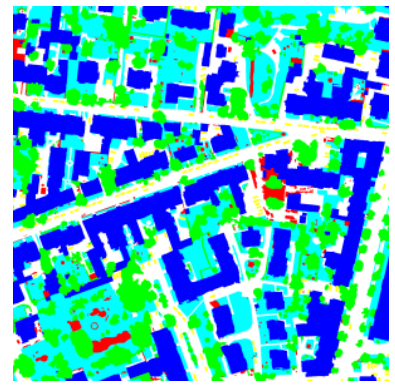

d) Ground truth
Figure 1. The Potsdam dataset contain: a) true colour aerial image (RGB and IR), b) DSM, c) NDSM and d) corresponding ground truth

This data contains 38 patches, patch $4 \_11$ is used in this paper which consists of the high-resolution ortho-rectified aerial image that has 4 channels: red, green, blue, and near-infrared bands. Digital surface model (DSM) is generated by dense image matching with pixel size $6000 \times 6000$ at the spatial resolution of $5 \mathrm{~cm}$. The ground truth labels are obtained by manual labelling (Sun, Zhang et al. 2018). In this paper NDSM is derived using an automatic filtering (Pahlavani, Amini Amirkolaee et al. 2017). Figure 1 illustrates this dataset.

\section{METHODOLOGY AND EXPERIMENTS}

Extraction of urban buildings with different colours, structures and textures is difficult for different methods, even the objectbased ones, to obtain a satisfactory result. Figure 2 shows the overall structure of proposed method for detection and extraction of buildings. To describe the proposed method, at first the study area was introduced and the suitable data were selected in Section 2. Then the procedure of segmentation is described in Section 3.1 and effective features are extracted in Section 3.2. Collecting train and test data is described in Section 3.3, then rules are produced using decision tree algorithm in Section 3.4. Finally according to the obtained rules, an object and rule-based classification methodology is applied to the dataset (Section 3.5) and is evaluated (Section 3.6).

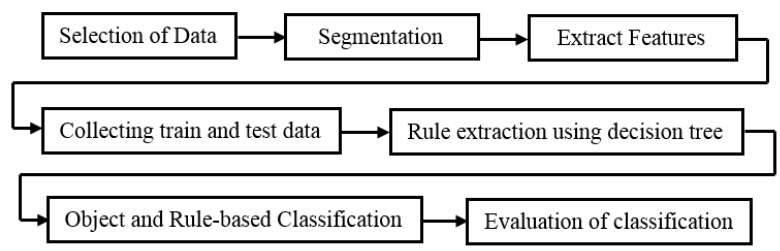

Figure 2. Flowchart of the proposed methodology

\subsection{Segmentation}

Image segmentation is the process of partitioning a digital image into a sets of pixels (segments, also known as super pixels). This process is the first step in object-based image analysis and its accuracy affects the overall result ( $\mathrm{Li}$, Zhang et al. 2018). The multiresolution segmentation algorithm is probably the most popular one for the purpose of the delineation of relatively homogeneous and meaningful objects. This procedure minimizes the average heterogeneity and maximizes their respective homogeneity for a given number of image objects, (Li, Zhang et al. 2018). The performance of segmentation is controlled by the user defined parameters which are scale, shape and compactness. Users have to repeatedly select a set of segmentation parameters and test them through a trial-and-error process, until a satisfied segmentation result is achieved (Tong, Maxwell et al. 2012).

After several experiments, appropriate segmentation parameters were determined. They are set as $30,0.5$ and 0.5 respectively. The visual results of the data in two scales are depicted in Figure 1.
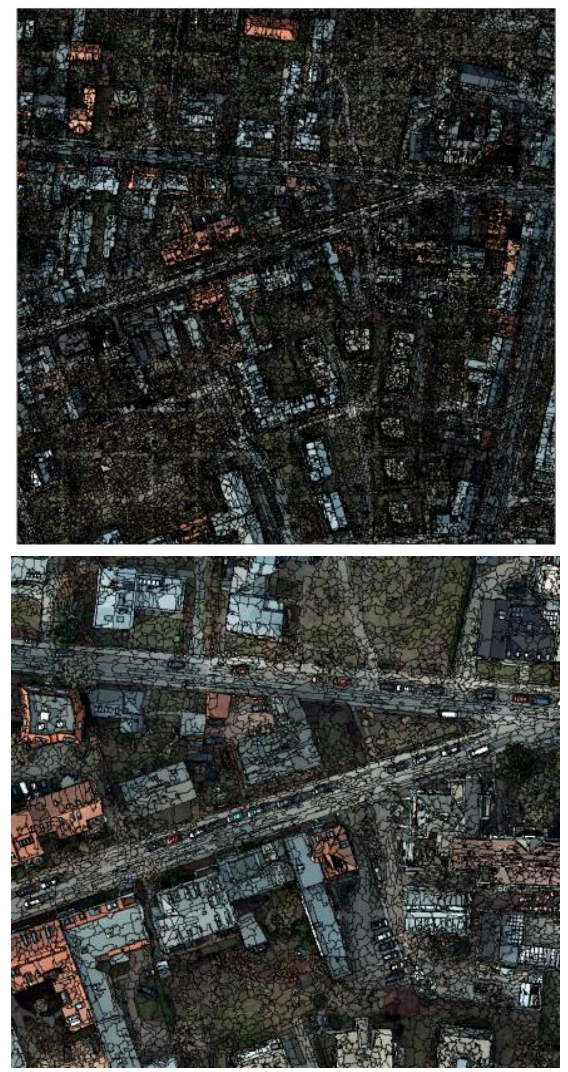

Figure 1. Multiresolution segmentation results in two scales.

\subsection{Extraction of features}

After the segmentation process, spectral and spatial features of the images are extracted. Indeed good classification system is conductive to selecting appropriate features or combinations of feature (Li, Zhang et al. 2018). Spectral features are related to all values of a segment, including metrics for maximum and minimum values of pixels or texture properties, while spatial features measure the shapes of objects-based, such as length and width.

In Table 1 and

3.3 Collecting train and test data 
Here is two classes: Building and Not_building. To collect train and test data the ground truth image is used. Some segments are selected randomly to label the training data and the corresponding feature values in that segments are selected as training values.

In this research, about $5 \%$ of pixels in each area were chosen as the training inputs and the rest (about 95\%) were selected as testing samples.

Table 2 common spectral and spatial features extracted from the segments are listed, respectively. for more information about features visit (Körting, Fonseca et al. 2013).

Table 1. Spectral features extracted from the segments

\begin{tabular}{|c|c|}
\hline Name & Description \\
\hline Amplitude & $\begin{array}{l}\text { Defines the maximum pixel value } \\
\text { minus the minimum pixel value. }\end{array}$ \\
\hline Dissimilarity & $\begin{array}{l}\text { Measures how different are the } \\
\text { Gary Level Co-occurrence Matrix } \\
\text { (GLCM) elements. }\end{array}$ \\
\hline Entropy & $\begin{array}{l}\text { Measures the disorder in an image. } \\
\text { When the image is not uniform, } \\
\text { many GLCM elements have small } \\
\text { values, resulting in large entropy. }\end{array}$ \\
\hline Homogeneity & $\begin{array}{c}\text { Assumes higher values for smaller } \\
\text { differences in the GLCM. }\end{array}$ \\
\hline Mean & $\begin{array}{c}\text { Returns the average value for all } N \\
\text { pixels inside the region. }\end{array}$ \\
\hline Mode & $\begin{array}{l}\text { Returns the most occurring value } \\
\text { for all } N \text { pixels inside the region. }\end{array}$ \\
\hline $\begin{array}{l}\text { standard } \\
\text { deviation }\end{array}$ & $\begin{array}{l}\text { Returns the standard deviation of } \\
\text { all } N \text { pixels }\end{array}$ \\
\hline NDVI & $\begin{array}{l}\text { normalized difference vegetation } \\
\text { index }\end{array}$ \\
\hline SAVI & Soil-adjusted vegetation index \\
\hline DSM & $\begin{array}{l}\text { An elevation model that includes } \\
\text { the tops of buildings, trees, and } \\
\text { any other objects. }\end{array}$ \\
\hline NDSM & $\begin{array}{l}\text { difference between DSM and } \\
\text { Digital Terrain Model (DTM) }\end{array}$ \\
\hline
\end{tabular}

\subsection{Collecting train and test data}

Here is two classes: Building and Not_building. To collect train and test data the ground truth image is used. Some segments are selected randomly to label the training data and the corresponding feature values in that segments are selected as training values.

In this research, about $5 \%$ of pixels in each area were chosen as the training inputs and the rest (about 95\%) were selected as testing samples.

Table 2. Spatial features extracted from the segments

\begin{tabular}{|c|c|}
\hline Name & Description \\
\hline Angle & $\begin{array}{l}\text { Represents the main direction of } \\
\text { a region. It is retrieved by the } \\
\text { angle of the biggest radius of the } \\
\text { minimum circumscribing ellipse. }\end{array}$ \\
\hline Area & $\begin{array}{c}\text { Returns the area of the region. } \\
\text { When measured in pixels is } \\
\text { equal to } N \text {. }\end{array}$ \\
\hline Box area & $\begin{array}{l}\text { Returns the bounding box area } \\
\text { of a region, measured in pixels. }\end{array}$ \\
\hline Circle & $\begin{array}{l}\text { Relates the areas of the region } \\
\text { and the smallest } \\
\text { circumscribing circle. } R \text { stands } \\
\text { for maximum distance between } \\
\text { the centroid and all vertices. }\end{array}$ \\
\hline Elliptic fit & $\begin{array}{l}\text { Finds the minimum } \\
\text { circumscribing ellipse to the } \\
\text { region and returns the ratio } \\
\text { between the area and } \\
\text { the ellipse area. }\end{array}$ \\
\hline $\begin{array}{c}\text { Fractal } \\
\text { dimension }\end{array}$ & $\begin{array}{c}\text { Returns the fractal dimension of } \\
\text { a region. }\end{array}$ \\
\hline $\begin{array}{l}\text { Gyration } \\
\text { radius }\end{array}$ & $\begin{array}{l}\text { Equals the average distance } \\
\text { between each pixel position in } \\
\text { one region and its centroid. } \\
\text { Smaller } \\
\text { values stand for regions similar } \\
\text { to a circle. }\end{array}$ \\
\hline Perimeter & $\begin{array}{l}\text { It is the amount of pixels in the } \\
\text { region's border }\end{array}$ \\
\hline $\begin{array}{l}\text { Perimeter area } \\
\text { ratio }\end{array}$ & $\begin{array}{l}\text { Is the ration between the regions } \\
\text { are and the minimum rectangle } \\
\text { outside the region. Higher values } \\
\text { stand for regions similar to a } \\
\text { rectangle. }\end{array}$ \\
\hline Rectangular fit & $\begin{array}{l}\text { Is the ration between the regions } \\
\text { are and the minimum rectangle } \\
\text { outside the region. Higher values } \\
\text { stand for regions similar to a } \\
\text { rectangle. }\end{array}$ \\
\hline Width & $\begin{array}{l}\text { It is the width of the region's } \\
\text { bounding box. }\end{array}$ \\
\hline
\end{tabular}

\subsection{Rule Extraction}

Decision tree, one of popular classification methodologies, is capable of classifying a dataset, which is defined by several features. In classification a particular feature begins at the root node, and the appropriate branch to a descendent node is followed. This procedure is repeated until a leaf node is reached, which has a class label. It trying to create a simple and compact tree with few nodes and deciding which attribute 
should be used to split the training data set at each node such that it can create a simple model that explains the data appropriately. The $\mathrm{C} 4.5$ decision tree algorithm is considered to be a robust, efficient and accurate algorithm capable of generating simple and effective decision trees from which classification rules can be extracted (Ren, Zargham et al. 2006). C4.5 adopts entropy impurity and automatically selects the attribute that provides the highest information gain ratio as the splitting attribute such that the splitting attribute can partition the data set with the best improvement on purity. The equations for computing gain ratio are as follows (Han and Kamber 2006, Nugroho, Adji et al. 2018):

$$
\operatorname{GainRatio}(A)=\frac{\operatorname{Gain}(A)}{\operatorname{SplitInfo}_{A}(D)}
$$

where

$$
\begin{aligned}
& A=\text { refer to an attribute } \\
& D=\text { refer to a dataset }
\end{aligned}
$$$$
\text { Gain }(A)=\text { gain of each attribute }
$$$$
\text { SplitInfo } \mathrm{A}(\mathrm{D})=\text { split attribute information }
$$

SplitInfo A (D) can be calculated as follows:

$$
\operatorname{SplitInfo}_{A}(D)=-\sum_{j=1}^{v} \frac{\left|D_{j}\right|}{|D|} \times \log _{2}\left(\frac{\left|D_{j}\right|}{|D|}\right)
$$

where

$$
\begin{aligned}
& v=\text { the number of classes } \\
& D=\text { the number of frequencies of the data instance } \\
& D_{j}=\text { the number of frequencies in the } j \text {-th attribute }
\end{aligned}
$$

The equation for finding the gain is as follows:

$$
\operatorname{Gain}(A)=\operatorname{Info}(D)-\operatorname{Info}_{A}(D)
$$

where

Info (D) $=$ the expected information needed to classify a tuple in D.

$\operatorname{Info}_{\mathrm{A}}(\mathrm{D})=$ the expected information required to classify a tuple from $\mathrm{D}$ based on the partitioning by attribute $\mathrm{A}$.

Info(D) and InfoA (D) have been expressed in equations (4) and (5), respectively:

$$
\begin{gathered}
\operatorname{Info}(D)=-\sum_{i=1}^{m} p_{i} \log _{2}\left(p_{i}\right) \\
\operatorname{Info}_{A}(D)=\sum_{j=1}^{v} \frac{\left|D_{j}\right|}{|D|} \times \operatorname{Info}\left(D_{j}\right)
\end{gathered}
$$

\begin{tabular}{|c|c|}
\hline Rule No. & Rule Description \\
\hline Rule 1 & $\begin{array}{l}\text { IF NDSM> } 28.786 \& \text { NDVI }>0.103 \& \text { Shape_Index }>0.097 \\
\text { then Not building }\end{array}$ \\
\hline Rule 2 & $\begin{array}{l}\text { IF NDSM }>28.786 \& \text { NDVI }>0.103 \& \text { Shape_Index } \leq 0.097 \& \& \\
\text { NDVI }>0.260 \text { then Not building }\end{array}$ \\
\hline Rule 3 & $\begin{array}{l}\text { IF NDSM }>28.786 \& \text { NDVI }>0.103 \& \text { Shape_Index } \leq 0.097 \\
\& \text { NDVI } \leq 0.260 \text { then Building }\end{array}$ \\
\hline Rule 4 & $\begin{array}{l}\text { IF NDSM }>28.786 \& \text { NDVI } \leq 0.103 \& \text { DSM }>44.258 \text { then } \\
\text { Building }\end{array}$ \\
\hline Rule 5 & $\begin{array}{l}\text { IF NDSM }>28.786 \& \text { NDVI } \leq 0.103 \& \text { DSM } \leq 44.258 \& \text { \& } \\
\text { Sum_Blue }>0.090 \& \text { DSM }>39.322 \text { then Building }\end{array}$ \\
\hline
\end{tabular}

where

$p_{i}=$ the nonzero probability that an arbitrary tuple in $D$ belongs to class $C$.

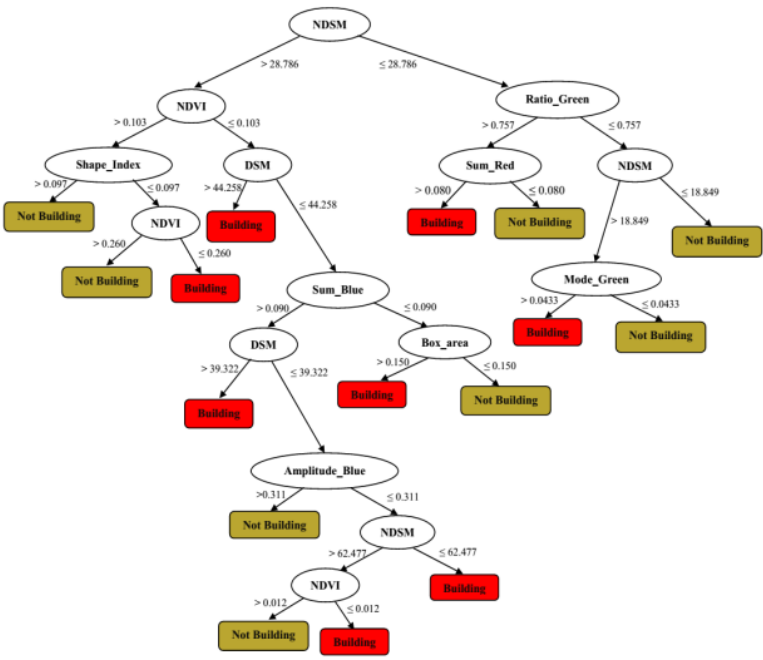

Figure 2. C4.5 decision tree classification model

Table 3. Extracted rules from decision tree

IF NDSM $>28.786 \&$ NDVI $\leq 0.103 \&$ \& $\mathrm{DSM} \leq 44.258 \&$ Sum Blue $>0.090 \&$ DSM $\leq 39.322 \&$ Amplitude Blue $>0.311$ then Not building Sum_Blue $>0.090 \& \mathrm{DSM} \leq 39.322 \&$ Amplitude_Blue $\leq 0.311$ $\&$ NDSM $>62.477 \&$ NDVI $>0.012$ then Not building Sum Blue $>0.090 \& \mathrm{DSM} \leq 39.322 \&$ Amplitude Blue $\leq 0.311$ $\&$ NDSM $>62.477 \&$ NDVI $\leq 0.012$ then Building Sum Blue $>0.090 \&$ DSM $\leq 39.322 \&$ Amplitude Blue $\leq 0.311$ $\& \mathrm{NDSM} \leq 62.477$ then Building

Rule $10 \quad$ IF NDSM $>28.786 \&$ NDVI $\leq 0.103 \&$ \& $\mathrm{DSM} \leq 44.258 \&$ Sum_Blue $\leq 0.090 \&$ Box_area $>0.150$ then Building

Rule 11 IF NDSM $\leq 28.786$ \& Ratio Green $>0.757$ \& Sum Green> 0.080 then Building

IF NDSM $\leq 28.786 \&$ Ratio_Green $>0.757 \&$ Sum Red $\leq 0.080$ then Not building

IF NDSM $\leq 28.786 \&$ Ratio Green $\leq 0.757 \&$ NDSM $>18.849 \&$ Mode_Green $>0.433$ then Building

IF NDSM $\leq 28.786 \&$ Ratio_Green $\leq 0.757 \&$ NDSM $>18.849 \&$ Mode_Green $\leq 0.433$ then Not building

Rule $15 \quad$ IF NDSM $\leq 28.786 \&$ Ratio_Green $\leq 0.757 \&$ NDSM $\leq 18.849$ then Not building

The decision tree classification model is constructed from training data and is illustrated in Figure 2 then the generated rules are listed in Table 3. 


\subsection{Object and rule-based classification}

According to the obtained rules the dataset is classified in two classes, Building (with red colour) and Not_building. The result of classification is shown in Figure 3.

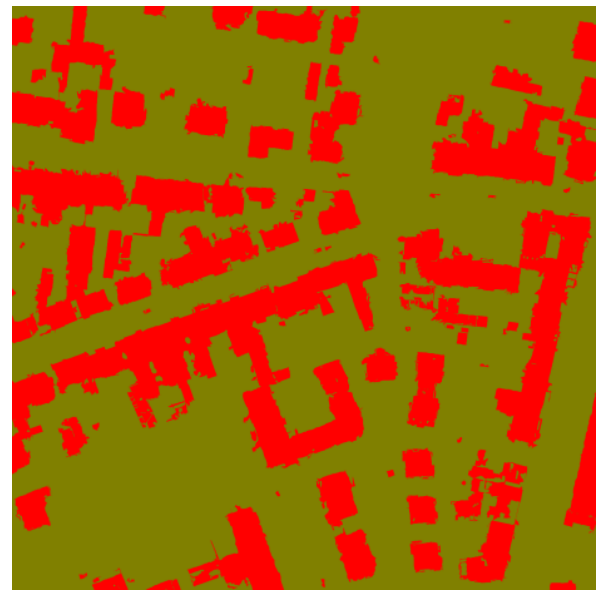

Figure 3. Extracted building from rule-based classification

\subsection{Evaluation of classification}

In the last step, the classification results are evaluated by using the overall accuracy and recall of each class. The overall accuracy of the classification was $96.43 \%$, the recall of Building class is $93.84 \%$ and recall of Not_building class is $99.04 \%$ which seem that the results are reasonable.

\section{CONCLUSION}

In this paper, an automatic framework to perform building extraction in object-based classification with remote sensing imagery is presented. The major contribution of this work is to avoid expert's role in generating rules by using a decision tree algorithm therefore the producer of defining appropriate rules become easier and more accurate.

\section{REFERENCES}

Ahmadi, S., M. V. Zoej, H. Ebadi, H. A. Moghaddam, A. Mohammadzadeh, 2010. "Automatic urban building boundary extraction from high resolution aerial images using an innovative model of active contours." International Journal of Applied Earth Observation and Geoinformation 12(3): 150-157.

Akçay, H. G., S. Aksoy, 2010. Building detection using directional spatial constraints. 2010 IEEE International Geoscience and Remote Sensing Symposium.

Arvor, D., N. Saint-Geours, S. Dupuy, S. Andrés, L. Durieux, 2013. Identifying optimal classification rules for geographic object-based image analysis.

Baatz, M., A. Schäpe, 2000. "Multiresolution segmentation: an optimization approach for high quality multi-scale image segmentation." In: Strobl, J., T. Blaschke, and G. Griesebner. "Angewandte Geographische Informationsverarbeitung XII." Wichmann, Heidelberg.: 12-23.

Benedek, C., X. Descombes, J. Zerubia, 2011. "Building development monitoring in multitemporal remotely sensed image pairs with stochastic birth-death dynamics." IEEE
Transactions on Pattern Analysis and Machine Intelligence 34(1): 33-50.

Benz, U. C., P. Hofmann, G. Willhauck, I. Lingenfelder, M. Heynen, 2004. "Multi-resolution, object-oriented fuzzy analysis of remote sensing data for GIS-ready information." ISPRS Journal of photogrammetry and remote sensing 58(3-4): 239258.

Bigdeli, B., Samadzadegan, F., Reinartz, P., 2013. "Classifier fusion of hyperspectral and LIDAR remote sensing data for improvement of land cover classifcation," International Archives of the Photogrammetry, Remote Sensing and Spatial Information Sciences, SMPR 2013, Tehran, Iran

Bigdeli, B., Pahlavani, P., 2016. "High resolution multisensor fusion of SAR, optical and LiDAR data based on crisp vs. fuzzy and feature vs. decision ensemble systems", International journal of applied earth observation and geoinformation 52(1): 126-136

Bigdeli, B., Pahlavani, P., 2017. "Quad-polarized synthetic aperture radar and multispectral data classification using classification and regression tree and support vector machinebased data fusion system." Journal of applied remote sensing 11(1): 016007

Bigdeli, B., Samadzadegan, F., Reinartz, P., 2014. "Feature grouping based multiple fuzzy classifier system for fusion of hyperspectral and LiDAR data." Journal of applied remote sensing 8(1): 083509

Blaschke, T., 2010. "Object based image analysis for remote sensing." ISPRS journal of photogrammetry and remote sensing 65(1): 2-16

Blaschke, T., C. Burnett, A. Pekkarinen, 2004. Image segmentation methods for object-based analysis and classification. Remote sensing image analysis: Including the spatial domain, Springer: 211-236.

Bouziani, M., K. Goita, D.-C. He, 2010. "Rule-based classification of a very high resolution image in an urban environment using multispectral segmentation guided by cartographic data." IEEE Transactions on Geoscience and Remote Sensing 48(8): 3198-3211.

Bruzzone, L., S. B. Serpico, 2000. "A technique for feature selection in multiclass problems." International Journal of Remote Sensing 21(3): 549-563.

Chen, J., H. Zheng, X. Lin, Y. Wu, M. Su, 2018. "A novel image segmentation method based on fast density clustering algorithm." Engineering Applications of Artificial Intelligence 73: 92-110.

Drăguţ, L., O. Csillik, C. Eisank, D. Tiede, 2014. "Automated parameterisation for multi-scale image segmentation on multiple layers." ISPRS Journal of Photogrammetry and Remote Sensing 88: 119-127.

Gao, Y., J. F. Mas, N. Kerle, J. A. Navarrete Pacheco, 2011. "Optimal region growing segmentation and its effect on classification accuracy." International journal of remote sensing 32(13): 3747-3763. 
Gavankar, N. L., S. K. Ghosh, 2019. "Object based building footprint detection from high resolution multispectral satellite image using K-means clustering algorithm and shape parameters." Geocarto International 34(6): 626-643.

Han, J., M. Kamber, 2006. "Data Mining: Concepts and Techniques."

Huang, H., F. Meng, S. Zhou, F. Jiang, G. Manogaran, 2019. "Brain image segmentation based on FCM clustering algorithm and rough set." IEEE Access 7: 12386-12396.

Jumlesha, S., S. Babu, C. Satyanarayana, M. Srinivas, M. Ramanaiah, S. Kumar, 2012. "Automatic urban feature extraction using mathematical morphology." Int J Eng Res Appl 2(3): 221-225.

Körting, T. S., L. M. G. Fonseca, G. Câmara, 2013. "GeoDMA - Geographic data mining analyst." Computers \& Geosciences 57: 133-145.

Lhomme, S., D. C. He, C. Weber, D. Morin, 2009. "A new approach to building identification from very-high-spatial-resolution images." International Journal of Remote Sensing 30(5): 1341-1354.

Li, R., T. Zhang, R. Geng, L. Wang, 2018. "Combining High Spatial Resolution Optical And Lidar Data for Object-Based Image Classification." International Archives of the Photogrammetry, Remote Sensing and Spatial Information Sciences 42: 3 .

Liu, Z., J. Wang, W. Liu, 2005. Building extraction from high resolution imagery based on multi-scale object oriented classification and probabilistic Hough transform. Proceedings. 2005 IEEE International Geoscience and Remote Sensing Symposium, 2005. IGARSS'05., IEEE.

Ming, D., J. Li, J. Wang, M. Zhang, 2015. "Scale parameter selection by spatial statistics for GeOBIA: Using mean-shift based multi-scale segmentation as an example." ISPRS Journal of Photogrammetry and Remote Sensing 106: 28-41.

Nugroho, H. W., T. B. Adji, N. A. Setiawan, 2018. Performance Improvement of C4. 5 Algorithm using Difference Values Nodes in Decision Tree. 2018 6th International Conference on Cyber and IT Service Management (CITSM), IEEE.

Pahlavani, P., H. Amini Amirkolaee, B. Bigdeli, 2017. "3D reconstruction of buildings from LiDAR data considering various types of roof structures." International journal of remote sensing 38(5): 1451-1482.

Pahlavani, P., Bigdeli, B., 2017. "A mutual informationDempster-Shafer based decision ensemble system for land cover classification of hyperspectral data." Frontiers of Earth Science, 11(4): 774-783.

Pang, S., X. Hu, Z. Wang, Y. Lu, 2014. "Object-based analysis of airborne LiDAR data for building change detection." Remote Sensing 6(11): 10733-10749.

Ren, N., M. Zargham, S. Rahimi, 2006. "A decision tree-based classification approach to rule extraction for security analysis."
International Journal of Information Technology \& Decision Making 5(01): 227-240.

Shi, W., Z. Mao, J. Liu, 2019. "Building area extraction from the high spatial resolution remote sensing imagery." Earth Science Informatics 12(1): 19-29.

Stankov, K., D.-C. He, 2014. "Detection of buildings in multispectral very high spatial resolution images using the percentage occupancy hit-or-miss transform." IEEE Journal of Selected Topics in Applied Earth Observations and Remote Sensing 7(10): 4069-4080.

Sun, Y., X. Zhang, X. Zhao, Q. Xin, 2018. "Extracting building boundaries from high resolution optical images and LiDAR data by integrating the convolutional neural network and the active contour model." Remote Sensing 10(9): 1459.

Teo, T.-A., L.-C. Chen, 2004. Object-based building detection from LiDAR data and high resolution satellite imagery. Proceedings of the 25th Asian Conference on Remote Sensing.

Tong, H., T. Maxwell, Y. Zhang, V. Dey, 2012. "A supervised and fuzzy-based approach to determine optimal multi-resolution image segmentation parameters." Photogrammetric Engineering \& Remote Sensing 78(10): 1029-1044.

Toure, S., D. Stow, H.-c. Shih, L. Coulter, J. Weeks, R. Engstrom, A. Sandborn, 2016. "An object-based temporal inversion approach to urban land use change analysis." Remote Sensing Letters 7(5): 503-512.

Walter, V., 2004. "Object-based classification of remote sensing data for change detection." ISPRS Journal of Photogrammetry and Remote Sensing 58(3): 225-238.

Xu, Y., L. Wu, Z. Xie, Z. Chen, 2018. "Building extraction in very high resolution remote sensing imagery using deep learning and guided filters." Remote Sensing 10(1): 144.

Yu, Q., P. Gong, N. Clinton, G. Biging, M. Kelly and D.

Schirokauer, 2006. "Object-based detailed vegetation classification with airborne high spatial resolution remote sensing imagery." Photogrammetric Engineering \& Remote Sensing 72(7): 799-811.

Zhang, R., D. Zhu, 2011. "Study of land cover classification based on knowledge rules using high-resolution remote sensing images." Expert Systems with Applications 38(4): 3647-3652.

Zhou, W., A. Troy, M. Grove, 2008. "Object-based land cover classification and change analysis in the Baltimore metropolitan area using multitemporal high resolution remote sensing data." Sensors 8(3): 1613-1636.

Ziaei, Z., B. Pradhan, S. B. Mansor, 2014. "A rule-based parameter aided with object-based classification approach for extraction of building and roads from WorldView-2 images." Geocarto International 29(5): 554-569. 